\title{
Oncolytic viruses: overcoming translational challenges
}

\author{
Jordi Martinez-Quintanilla, ${ }^{1}$ Ivan Seah, ${ }^{1}$ Melissa Chua, ${ }^{1,2}$ and Khalid Shah ${ }^{1,2,3}$ \\ 'Center for Stem Cell Therapeutics and Imaging and ${ }^{2}$ Department of Neurosurgery, Brigham and Women's Hospital, Harvard Medical School, Boston, Massachusetts, USA. ${ }^{3}$ Harvard Stem Cell Institute, \\ Harvard University, Cambridge, Massachusetts, USA.
}

\begin{abstract}
Oncolytic virotherapy (OVT) is a promising approach in which WT or engineered viruses selectively replicate and destroy tumor cells while sparing normal ones. In the last two decades, different oncolytic viruses (OVs) have been modified and tested in a number of preclinical studies, some of which have led to clinical trials in cancer patients. These clinical trials have revealed several critical limitations with regard to viral delivery, spread, resistance, and antiviral immunity. Here, we focus on promising research strategies that have been developed to overcome the aforementioned obstacles. Such strategies include engineering $\mathrm{OVs}$ to target a broad spectrum of tumor cells while evading the immune system, developing unique delivery mechanisms, combining other immunotherapeutic agents with OVT, and using clinically translatable mouse tumor models to potentially translate OVT more readily into clinical settings.
\end{abstract}

\section{Introduction}

Oncolytic virotherapy (OVT) is a promising approach in which viruses selectively replicate in and destroy tumor cells while sparing normal ones. The biological amplification of oncolytic viruses (OVs) by viral replication in the tumor cells is one of the major advantages of OVTs over other cancer therapies (1). To increase their utility as anticancer agents, OVs generally are engineered to further increase their antitumor specificity, safety, immunogenicity, and potency (2). OVs have two main mechanisms of action: first, the direct infection of cancer cells and associated endothelial cells (ECs) that results in oncolysis of these cell types in the tumor microenvironment (TME); and second, antitumor immunity elicited by the $\mathrm{OV}$ as a consequence of improved antigen cross-priming and recruitment of immune cells into the TME $(3,4)$.

Clinical trials have extensively demonstrated the tolerability of OVs in patients (5) and in some cases have shown moderate OV-mediated antitumor efficacy $(6,7)$, such as the recent phase III clinical trials in patients with advanced or metastatic melanoma treated with talimogene laherparepvec (T-VEC) (ref. 8 and Table 1). However, clinical trials with OVs still have not shown robust antitumor efficacy, especially with oncolytic virus monotherapy. In this Review, we provide an overview of the critical limitations of OVs that have hampered their progress in clinics for therapeutic use and summarize innovative research strategies that have been explored to overcome these obstacles.

\section{Enhancing the efficacy of OVTs}

During the last decade, development of a new generation of therapies based on OVs capable of inducing tumor remissions in

Conflict of interest: KS owns equity in and is a member of the board of directors of AMASA Technologies Inc., a company developing cell-based therapies for cancer. KS's interests were reviewed and are managed by Brigham and Women's Hospital and Partners HealthCare in accordance with their conflict of interest policies.

Copyright: (๑) 2019 American Society for Clinical Investigation

Reference information: J Clin Invest. 2019;129(4):1407-1418.

https://doi.org/10.1172/JCI122287. preclinical models has been extensively explored (9-11). A perspective on some of the prevalent strategies exploring different avenues to enhance efficacy of OVT is given below.

Enhancing intratumoral viral spread. Early clinical trials showed that although OVs accessed tumor cells after intratumoral or i.v. administration, viral replication was generally transient and occurred in localized areas of the tumor, resulting in suboptimal antitumor efficacy $(12,13)$. Subsequent preclinical studies demonstrated that the main sources of physical barriers to OVs were the extracellular matrix (ECM) proteins, polysaccharides, tumor-associated fibroblasts, inflammatory cells, and high interstitial fluid pressure in the tumor mass $(14,15)$. Hyaluronic acid (HA) and collagen are major components of ECM, and previous preclinical studies have shown that degradation of HA by a proteolytic enzyme, hyaluronidase, reduces interstitial fluid pressure, permitting anticancer agents to reach breast cancer cells $(16,17)$. Consequently, ICOVIR17, an armed oncolytic adenovirus expressing hyaluronidase $\mathrm{PH} 2 \mathrm{O}$, has been shown to degrade the ECM and enhance spread into the solid tumor mass in xenograft mouse models, ultimately improving the outcomes in treated mice (16). We have previously shown that ICOVIR17 degrades the HA in glioblastoma (GBM) tumors, leading to an enhanced distribution of ICOVIR17 within the tumor and a subsequent significant increase in tumor cell death in mouse tumor models of GBM (ref. 18 and Figure 1A). VCN-01, an ICOVIR17 version with improved tumor targeting (19), has shown therapeutic effects in pediatric osteosarcoma (20) and brain tumor mouse models (21) and is currently being tested in two phase I clinical trials in advanced solid tumors (Table 1). In a separate preclinical study, vaccinia virus (VV) GLV-1h255, engineered to express metalloproteinase 9, led to degradation of collagen IV in the tumor, facilitating intratumoral viral dissemination and resulting in tumor regression (22). Degradation of ECM by relaxinexpressing OVs has also shown increased viral distribution and inhibition of tumor growth (23) as well as tumor sensitization to chemo(24) and radiotherapy (25) in animal tumor models. OVs expressing decorin, an inhibitor of TGF- $\beta$, have also been tested in mouse models of lung and bone metastasis $(26,27)$. Systemic administration of oncolytic adenovirus expressing decorin in an immune-competent 
Table 1. Clinical trials with OVs in last 3 years

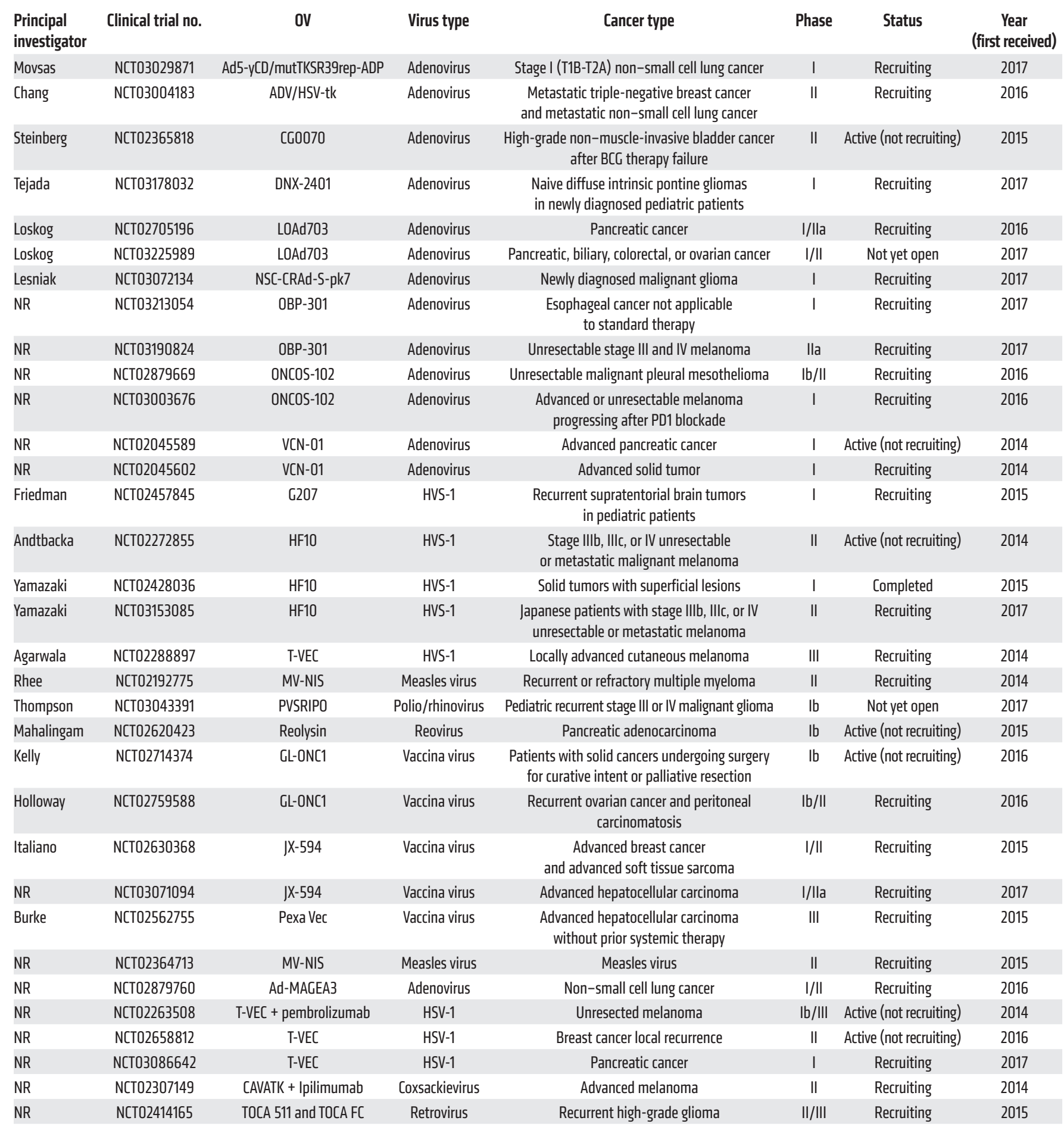

Because of space constraints, we have included selected examples of the most relevant clinical trials in last 3 years. We apologize to investigators whose work has not been included. NR, not reported.

mouse model of lung metastasis modulated the antitumor inflammatory and immune responses via activation of $\mathrm{CD}^{+} \mathrm{T}$ cells (26).

There are contradictory findings with regards to the function of ECM in tumor metastasis. Some studies have shown that ECM promotes tumor metastasis (28), whereas other stud- ies implicate the degradation of HA in cancer progression and metastasis (29), thus raising concerns about the safety of OVs expressing ECM-degrading factors. However, OVs expressing ECM-degrading enzymes have been engineered to express the transgenes in the late phase of viral replication, resulting in a 
A

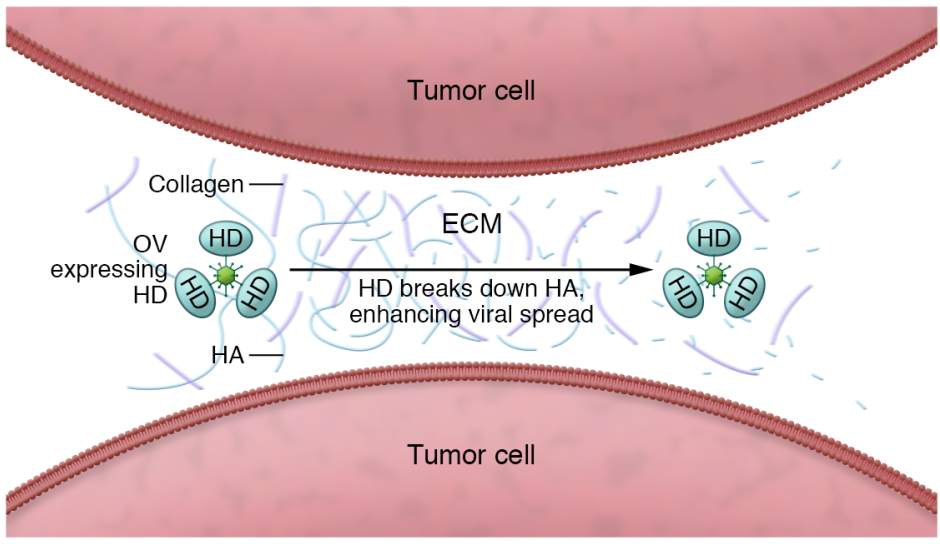

B

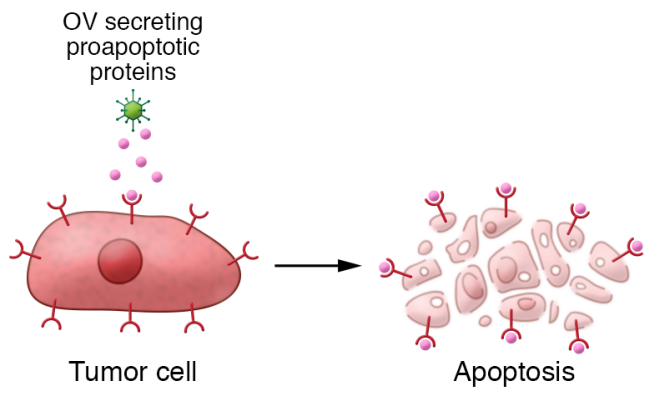

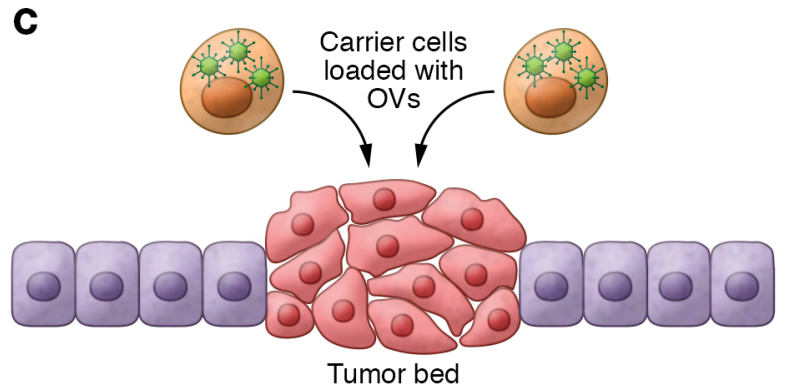

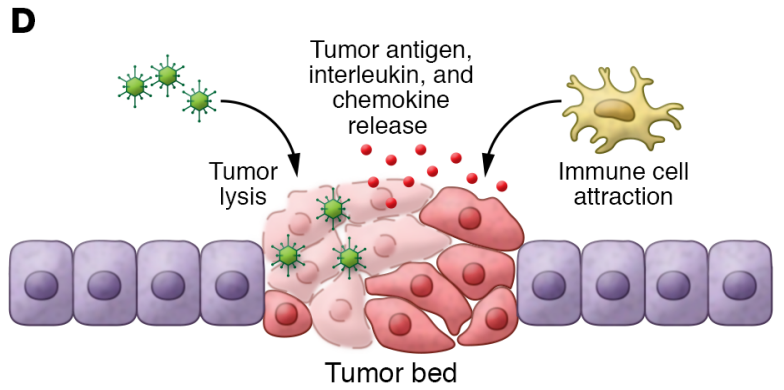

Figure 1. Strategies to circumvent the obstacles observed in clinical trials using OVs. (A) Enhancing intratumor viral spread. OVs engineered to express hyaluronidase (HD) are able to break down HA in the ECM, enhancing the ease of intratumor spread of the OV. (B) Sensitize tumor cells to OV therapy. OVs engineered to secrete proapoptotic proteins revert tumor resistance to OV therapies. (C) Optimizing OV delivery. Carrier cells protect OVs from the immune system and increase tumor targeting of OVs. (D) OV-mediated immunotherapy. OV-mediated oncolysis boosts the immune system response against tumor cells, improving overall therapeutic response.

localized degradation of the ECM around OV-infected cells. This strategy minimizes the possibility of exposing uninfected tumor cells to ECM degradation. OVs have also been engineered to express hyper-fusogenic envelope glycoproteins to improve intratumoral viral spread. Preclinical studies have shown that the expression of these proteins in tumor cells induces cellto-cell fusion, thus allowing the virus to spread without being exposed to the intracellular spaces (30-32).

Strategies to sensitize tumor cells to OVT. Many studies have shown that advanced tumors have a tremendous capacity to evolve and develop resistance to a wide variety of therapeutic agents (33). OVs attack tumor cells in multiple different ways, and therefore, tumors acquire resistance less frequently compared with other therapies. However, previous preclinical studies have shown that tumor cell lines have variable levels of sensitivity to OV-mediated killing and can acquire resistance to OVs $(34,35)$. Continuous exposure of tumor cells to reovirus can lead to resistance. This resistance is mediated by increased protein kinase R phosphorylation, which itself contributes to diminished viral replication potential, but also decreases activity of endosomal cathepsin B, which is required for efficient reoviral entry and activation (34). Another study has shown that tumor cells continuously exposed to oncolytic adenovirus can acquire resistance by blocking the lytic phase of the OV (35). To overcome this resistance, several groups have demonstrated that PI3K inhibitors (36), proteasome inhibitors (36), or rapamycin (37) sensitize OV-resistant tumors to virother- apy. Proapoptotic TNF apoptosis-inducing ligand (TRAIL) has been shown to induce apoptosis in a wide range of human cancer cell lines without significant cytotoxicity toward normal cells (38). We have previously shown that oncolytic herpes simplex virus (oHSV) engineered to express a secretable and potent variant of proapoptotic TRAIL (oHSV-TRAIL) was able to target tumor cells resistant to both TRAIL and oHSV by altering cell proliferation pathways and activating caspase-mediated cell death pathways (refs. 39, 40 and Figure 1B). Similar findings were reported with an oncolytic adenovirus-TRAIL combination in a multiple myeloma mouse model (36). Although previous findings had suggested that TRAIL may have potential liver toxicity after systemic administration (41), localized delivery of TRAIL via OVs has been shown to have limited toxicity $(39,40)$.

Circumventing antiviral immunity. A substantial proportion of the human population has already been exposed to OVs and thus presents with preexisting humoral and cellular immunity against many of the OVs currently undergoing clinical development, including adenovirus (42), reovirus (43), VV (44), and measles virus (MV) (45). Consequently, OVs administered into the bloodstream are usually neutralized by antibodies, blood cells, complement, and antiviral cytokines (46) and are cleared by phagocytes in the liver and spleen before they reach the tumor mass (47). Rapid OV elimination from circulation following its systemic administration in patients contributes to the promising safety profile of OVT. However, it also results in a reduced antitumor effect. One 
A

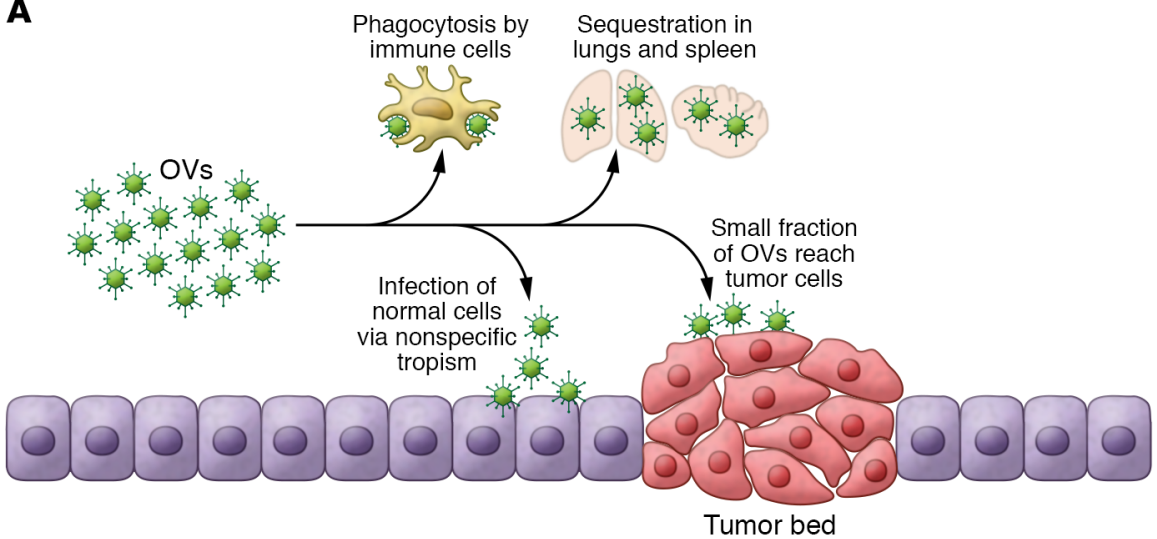

Figure 2. Optimizing OV delivery with SCs. (A) Intrinsic immunotherapy of OVs. OVs administered systemically are phagocytosed by immune cells before reaching tumor cells. Furthermore, some OVs infect normal cells via nonspecific tropism, and other OVs are sequestered in the lung and spleen. (B) SCs loaded with OVs migrate to intact tumors. SCs protect OVs from the immune cells and target them to the tumor sites. (C) SCs loaded with OVs migrate to debulked tumors. Tumor debulking releases tumor antigens and causes inflammation in the surrounding area, attracting SCs loaded with OVs to the remaining tumor deposits.

B
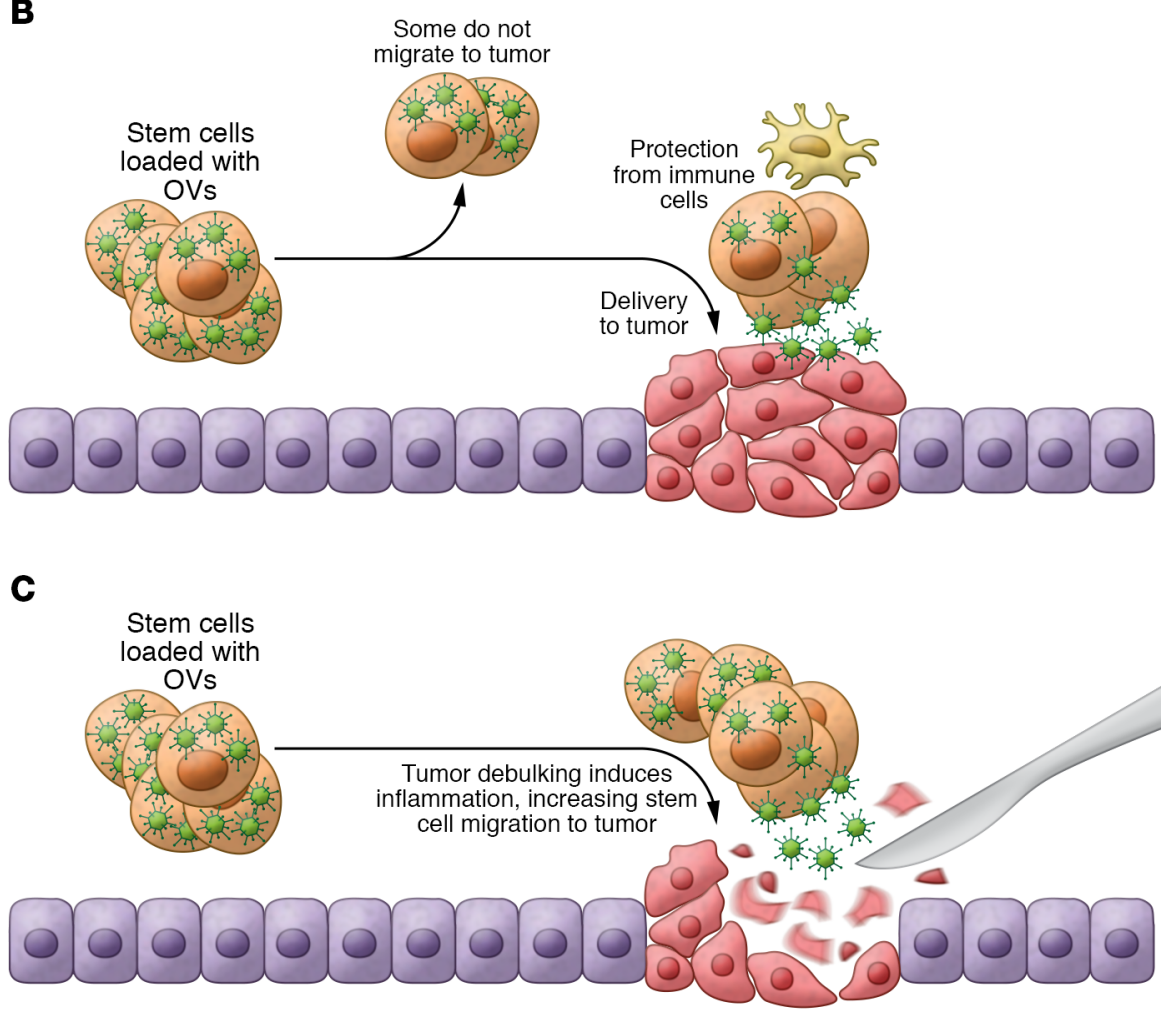

logical approach to circumventing antiviral immunity has been the coadministration of OVs with immunosuppressive drugs such as cyclophosphamide (48). Previous studies have shown that four daily doses of cyclophosphamide combined with MV or vesicular stomatitis virus (VSV) were able to significantly reduce antiviral antibody titers in mice, thus allowing effective repeated doses of OVs (49). Different viral families and serotypes within the same family can trigger differential immune and inflammatory responses. Several strategies have been developed to circumvent this, including using low-seroprevalent OVs, molecular engineering of chimeric OVs, and switching viral coat proteins. In the case of adenovirus, studies have shown that Ad5/35 (an Ad5 chimeric adenovirus expressing the fiber proteins of Ad35) reduces toxicity and limits the induction of inflammatory cytokines in murine and nonhuman primate animal models (50). In the case of MV, where serotype switching is not an option, the immunodominant epitopes of the viral surface glycoproteins have been modified by mutating key surface residues to reduce viral immunity (51). However, all these strategies have the potential to alter viral tropism (52). Other strategies that reduce viral neutralization are to polymer-coat the virus $(53,54)$ or use liposome-encapsulated OVs $(55)$, thereby blocking antibody recognition and extending the circulation times of the viruses in mice (53-55). However, these strategies are associated with a decrease in the binding of the virus to its cellular receptors, resulting in reduced tumor cell infection (53-55).

As mentioned previously, the promising safety profile achieved by systemic administration of OVs could be partially due to OV inactivation by preexisting innate and adaptive immunity. Therefore, toxicity studies should be carefully performed on strategies that reduce OV inactivation to determine whether these approaches modify the safety profile of OVT.

Optimizing OVT delivery. The efficacy of OVT and other cancer therapies depends heavily on the successful delivery of an antitumor agent in the tumor mass. Early clinical trials demonstrated 


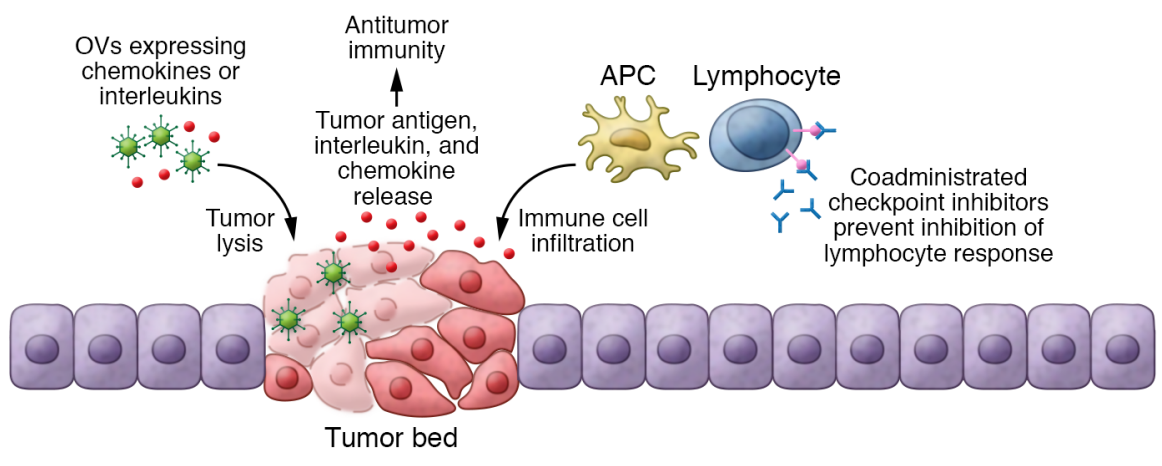

Figure 3. Oncolytic immunotherapy. OVs engineered to activate immune system release interleukins or chemokines after tumor cell infection, activating the immune response against the tumor. Coadministered checkpoint inhibitors prevent the inhibition of immune response, ensuring that immune cells are constantly activated against the tumor cells. APC, antigenpresenting cell. that intratumoral injection was the most efficient and safest way to administer OVs $(56,57)$. Recent preclinical studies in a Syrian hamster model suggested that innate immunity against the virus promoted the clearance of injected OVs after intratumoral administration, but did not affect antitumor efficacy (58). As a consequence, repeated intratumoral injections of OVs triggered a robust immune response against the virus, resulting in a therapeutic benefit (58). Since several malignancies, particularly metastatic disease, are inaccessible by direct injection, some groups have explored systemic administration of OVs, which would allow OVs to reach even distant metastases via the bloodstream (59). However, in this instance, a very low fraction of virus reaches the tumor in patients, because of viral neutralization in the blood stream; sequestration of the viruses through the fenestrated capillaries of the lung, spleen, and liver; and nonspecific tropism. Analysis of the tumor biopsies from patients treated with an oncolytic VV revealed that those receiving the highest dose $\left(10^{9} \mathrm{PFUs}\right)$ showed low amounts of virus within the tumor (60). These findings were supported by studies in mouse models, which detected a very low quantity of virus reaching the tumor mass after i.v. administration of $10^{9}$ PFUs of VSV (ref. 61 and Figure 2A). Preclinical strategies based on mutating the binding site sequence in capsid genes that interact with blood factors and scavenger receptors on macrophages have been shown to partially increase antitumor efficacy by extending viral circulation time and increasing tumor targeting after systemic administration of the virus (62). Other delivery routes such as intracranial $(63)$ and intraarterial $(64,65)$ have been explored, but with limited success. Although preclinical studies (66) and clinical trials (45) have shown promising results after i.p. administration of oncolytic MV in patients with recurrent ovarian cancer, this delivery route is limited to patients with peritoneal tumors, potentially reducing its efficacy in patients with metastatic cancer.

In recent years, cell-based carriers have emerged as the most promising delivery vehicles for OVTs. These carriers protect OVs from neutralizing antibodies, support viral replication and amplification, and specifically target OVs to the tumor mass (ref. 67 and Figure 1C). In general, OV carrier cells can be classified broadly into tumor cells, immune cells, and stem cells (SCs). To determine the most effective cell carrier for the delivery of OVs, it is essential to consider the susceptibility of the carrier cell to the virus and the kinetics of viral replication and release within carrier cell type as well as the kinetics of carrier cell trafficking from the site of injection to the tumor mass (68). Below, we discuss the available evidence supporting the use of each carrier cell type.
Tumor cells. Tumor cells proliferate readily and are therefore very permissive to virus infection and replication, resulting in a high viral production after infection (69). A growing body of evidence suggests that tumor progression at this stage may be enhanced by circulating cancer cells' ability to "self-seed," a process involving cell dissemination into the vascular system away from a primary or metastatic tumor, followed by the cells rehoming to the site of origin (70). Although the molecular mechanism that tumor cells use to target metastatic deposits is not well understood, it seems to be associated with the same receptor molecule repertoire (cell adhesion molecules, chemokine receptors, or integrin ligands) involved in the metastatic process. This cancer cell tropism to metastatic deposits is supported by several preclinical studies $(71,72)$, which suggest the potential of cancer cells to be used as OV carriers. While the innate tumorigenic potential of cancer cells raises safety concerns, previous studies have demonstrated that irradiated cells can serve as feeder layers for a certain time before dying (73). Using this time window, irradiating tumor cells just prior to OV infection has been shown to reduce their ability to grow without affecting tumor targeting or the production and release of OVs $(74,75)$. This poses a potential avenue for the safe use of tumor cells as OV carriers.

Immune cells. Immune cells naturally circulate in a systemic way and specifically migrate to and recognize tumor cells (76). Among the immune cell subsets, $\mathrm{T}$ cells and monocytes/macrophages are the most promising carrier cells. Specifically, $\mathrm{T}$ cells loaded with VSV (77), reovirus (78), HSV (79), and Newcastle disease virus (80) have been delivered to tumors in mouse models. Previous studies have demonstrated that engineering $\mathrm{T}$ cells with chimeric antigen receptors (CARs) increases the delivery of VSV and VV to tumor cells and that CAR expression and function are not affected by the cell's infection with OV (81). Despite the efficient homing of $\mathrm{T}$ cells to tumors, carrier $\mathrm{T}$ cells have limited ability to amplify OVs (81), and moreover this clinical application is challenging and expensive. A few studies have shown that the viability of carrier $T$ cells can be significantly improved by attaching VSV to the membrane of $\mathrm{T}$ cells, allowing gradual release of oncolytic VSV into the tumor mass $(82,83)$.

Tumor-associated macrophages (TAMs) are immune cells that localize to hypoxic regions in the tumor mass $(84,85)$. Administration by i.v. of TAMs loaded with MV into mice that were bearing myeloma tumors resulted in myeloma cell infection and prolonged mouse survival (86). In another study, macrophages loaded with a hypoxia-regulated oncolytic adenovirus showed a 
synergistic therapeutic effect when combined with chemotherapy and radiotherapy in a metastatic mouse model of prostate cancer (87). Although the use of different immune cells to deliver OVs offers promise, the overall feasibility of employing them as carriers will require extensive study.

SCs. SC-based therapies are emerging as another promising strategy to treat cancer. Mesenchymal stem/stromal cells (MSCs) in particular have generated immense interest because they can be easily loaded with OVs $(88,89)$ and home to areas of inflammation and tissue injury in preclinical tumor models (90). In mouse models, MSC-mediated delivery of oncolytic adenovirus to GBM tumors (91) and lung and breast metastatic tumors (92) has demonstrated therapeutic efficacy. We have previously shown that MSCs loaded with oHSV or oncolytic adenovirus can deliver viral progeny to established GBM tumors, reducing tumor growth and increasing mouse survival rates (refs. 18, 93, and Figure 2B). Although a number of studies have demonstrated that MSCs loaded with OVs have better therapeutic efficacy than naked OVs, tumor-homing and biodistribution of MSC-loaded OVs via different routes of administration require more detailed investigation. A few studies have shown that SCs possess immunosuppressive properties (94-96), suggesting that they would not be ideal carriers for OV-mediated immune stimulation. However, recent studies have clarified that OV infection in SCs induces TLR 9 overexpression and activation of the NF- $\kappa \mathrm{B}$ pathway, leading to a specific cytokine secretion profile by SCs and generating a proinflammatory environment (97).

Approximately $75 \%$ of GBM patients undergo tumor debulking $(98,99)$, and we have shown that delivering human MSCs encapsulated in biodegradable synthetic ECM (sECM) and loaded with oHSV or its proapoptotic variant, oHSV-TRAIL, into the mouse GBM tumor resection cavity significantly increased survival rates (ref. 93 and Figure 2C). Previous studies have shown significant therapeutic efficacy of MSC-mediated ICOVIR17 delivery compared with direct injection in a mouse model of GBM resection (18). In another promising approach, we have recently shown that intracarotid artery-mediated delivery of MSC-oHSV, but not oHSV alone, was able to selectively target metastatic melanoma lesions in the brain (100). Other studies have demonstrated that intracranial administration of immortalized neural SCs (NSCs) loaded with a fiber-modified oncolytic adenovirus, CRAd-S-pk7, results in a significant survival improvement in xenograft models $(101,102)$. These preclinical studies have led to an ongoing phase 1 clinical trial investigating the therapeutic efficacy of immortalized NSC-CRAd-S-pk7 in patients with GBM tumors (Table 1). However, the ideal NSC carrier cells for clinical use should be autologous to avoid immune rejection. Recent studies have demonstrated that induced NSCs derived from human fibroblasts have tumor-homing capacity in preclinical settings and therefore offer potential use as OV carrier cells (103).

One of the main constraints of using cellular vehicles to deliver OVs is the toxicity of the viral progeny on carrier cells $(91,104$, 105), which ultimately reduces the viral delivery and distribution in and around the tumor mass (106). Therefore, increasing the viability of OV-infected SCs and controlling viral replication within the delivery vehicles are critical and have been studied in detail. Previous studies have demonstrated that the DNA synthesis inhib- itor, mimosine, temporarily arrested OV replication after NSC loading, allowing OV-loaded NSCs to migrate to a GBM tumor prior to viral-induced NSC lysis in mice (104). Additionally, the ROS inhibitor $N$-acetylcysteine amide reduced OV-mediated toxicity by preventing ROS-induced apoptosis in carrier cells without reducing viral progeny (107). In another study, EGFP flanked by FLP recombinase sequences was incorporated into the oncolytic adenoviral genome (108). Using this strategy, carrier cells could be engineered to express FLP-recombinase driven by a hypoxia promoter and loaded with proAd-GFP, allowing reactivation of the OV upon reaching hypoxic areas of the tumor site in mice.

Although SCs loaded with OV will ultimately be killed by the lytic cycle of the virus, it is difficult to ensure whether all carrier cells are infected with OVs or whether some cells escape virus-induced death. Additionally, although SCs have been administered to many patients without considerable side effects, the capacity of any carrier cell to acquire oncogenic mutations is a potential safety concern. Preclinical studies in our laboratory have shown that engineering MSC to express HSV-thymidine kinase allowed selective elimination of carrier cells by administration of the prodrug ganciclovir (109). Such studies add a safety parameter and offer the potential for translating SCs loaded with OVs into clinical settings.

Other OV delivery vehicles and strategies. Tumor microparticles (TMPs) (110) have been used to deliver OVs into tumor cells in immunocompetent mice and have been shown to overcome the nuclear membrane barrier, thus facilitating the entry of the OV into the nucleus and eliminating tumor cells after OV replication (111). For instance, the ultrasound-mediated delivery of microbubble carriers enabled effective delivery of OVs into the targeted cells (112) by increasing the replicating virus at the tumor site (113) as well as improving bioavailability and intratumoral biodistribution of OVs (114).

\section{OV-mediated immunotherapy}

Intrinsic immunotherapy of OVs. Recently, a number of preclinical and clinical studies have shown that OVs are capable of dramatically altering the TME immune landscape, disrupting immune tolerance to cancer cells and leading to improved antitumor activity alone or in combination with assorted immune modulators (refs. 115, 116 and Figure 1D). OV-mediated cell killing is the first in a series of events that culminates in the induction of a robust and long-lasting antitumor adaptive immune response (117). OV infection triggers immunogenic cell death characterized by the expression of damage- or pathogen-associated molecular patterns (DAMPs or PAMPs), which attract and activate DCs and innate immune cells, respectively, in the TME (118). Once at the tumor site, DCs engulf OV-infected cancer cells and capture tumorassociated antigen (TAA) for cross-presentation to naive CD8 ${ }^{+}$ $\mathrm{T}$ cells, priming them against tumor cells in the lymph nodes (119, 120). These tumor-specific $\mathrm{T}$ cells enter the bloodstream to reach the inflamed tumor site, where they exert their cytotoxic effect in the remaining cancer cells displaying TAAs. Compared with other immunotherapies that use specific TAA identification, OVs vaccinate against a patient's entire TAA repertoire (4).

In mouse tumor models, adenovirus-induced tumor oncolysis elicited specific $\mathrm{T}$ cell responses to a panel of putative neopitopes, whereas novel immune checkpoint inhibitor monotherapy 
failed to trigger such tumor-specific responses (121). In addition, preclinical studies have shown that adaptive antitumor immune responses primed by i.v. injection of reovirus were independent of tumor oncolysis, indicating that viral replication is not critical to inducing OV-mediated immunotherapeutic effects (122). In addition to tumor cells and immune cells, OVs target other subsets of cells present in the TME, such as cancer-associated fibroblasts (CAFs), ECs, and pericytes, thus aiding in the disruption of the TME structure. Previous studies have shown that OVs can infect and replicate in CAFs (123) and ECs (124), resulting in their elimination and subsequently increasing immune infiltration in the TME. Most preclinical studies have concluded that OVs have the potential to convert immunologically inert tumors into highly immune-reactive ones and have the ability to attack tumor malignancies in multiple different ways by targeting different subsets of TME cells and disrupting the tumor landscape (125). Indeed, OVs create an acute localized inflammatory response in the tumor that favors immune cell recruitment and activation and results in a therapeutic antitumor effect. Recent preclinical studies using TOCA 511, a retroviral-replicating vector that encodes a prodrug activator enzyme, cytosine deaminase, resulted in a therapeutic effect mediated by suicide gene therapy as well as antitumor immunity in metastatic colorectal, pancreatic, and GBM tumor mouse models (126-128). These promising results led to several clinical trials in patients with GBM tumors, including the ongoing phase III trials (Table 1).

Combination of OVs with immunomodulators. Clinical trials using OVs have highlighted that antitumor immunity, which is critical to achieving clinically relevant therapeutic efficacy, is strongly associated with antiviral immunity. This immunity represents a sizeable hurdle for OVT, since it promotes OV clearance prior to achieving therapeutic impact on the tumor mass (129). In recent years, several studies have provided insights into balancing antitumor and antiviral immunity. One of the most promising strategies is the OV-mediated expression of cytokines, such as GM-CSF, which results in the increased recruitment of DCs to the TME, thereby increasing antitumor immunity (44, 60, and 130-133). The most promising clinical trial to date has been performed with T-VEC (oHSV-GM-CSF) (134), an ICP34.5-/ICP47-oHSV that combines immune stimulation and oncolytic lysis. T-VEC was the first OVT approved by the US FDA for the treatment of inoperable melanoma $(135,136)$ based on the promising results of a phase III clinical trial (8). Clinical trials with T-VEC have demonstrated patient safety (137) and prolonged patient survival compared with GM-CSF alone in unresected stage IIIB-IV cutaneous head and neck melanoma (ref. 138 and Table 1). Recent clinical trials have sought to demonstrate the efficacy of T-VEC in other types of solid tumors, such as breast and pancreatic tumors (Table 1). A similar approach uses JX-594, a genetically engineered VV with a deletion in its growth factor, VGF, and transgene-driven expression of GM-CSF (139). Clinical trials with JX-594 have shown tumor regression in some patients with hepatocellular carcinoma, lung cancer, colorectal cancer, and melanoma in a phase I/II clinical trial (refs. 7, 140-142, and Table 1). Chemokine ligands such as CCL3, -5, -7, -19, and -20 have also been engineered into OVs to stimulate the activity of antigen-presenting cells and enhance tumor infiltration. These strategies have elicited significant anti- tumor immune responses in mouse models (refs. 143-147 and Figure 3). In other studies, OVs engineered with IL-12 and IL-15 induced proliferation and activated $\mathrm{NK}$ and $\mathrm{T}$ cells, elicited potent antitumor effects, and prolonged mouse survival (148-151). In a different approach, oncolytic adenoviruses were armed with soluble EGFR-targeting bispecific T cell-engager (BiTE) antibodies. Tumor cells infected with OVs secreted BiTEs that bound specifically to $\mathrm{CD}^{+} \mathrm{T}$ cells and $\mathrm{EGFP}^{+}$tumor cells, resulting in an increased persistence and accumulation of tumor-infiltrating $\mathrm{T}$ cells in a mouse model of lung cancer (152).

Previous studies have also explored strategies to tip the balance toward antitumor immunity by reducing antiviral immunity against the OVT. Specifically, this approach uses two different OVs: adenovirus followed by a therapeutic VV. In a Syrian hamster model, consecutive OV administration evoked immune system responses only against adenovirus, thus allowing the therapeutic VV to induce its antitumor effect. Furthermore, this strategy showed that the administration of two consecutive OVs can also boost the antitumor immune response (153). Another challenging strategy that favors antitumor immunity is based on designing OVs that turn into oncolytic vaccines by expressing highly specific tumor antigens. As such, VSV and VV have been engineered to express human papilloma virus oncogene E7 (VSV-E7) (154), human dopachrome tautomerase (VSV-hDCT) (155), or human oncofetal antigen 5T4 (VV-h5T4) (156). These therapeutic oncolytic vaccines generated antigen-specific $\mathrm{CD} 4^{+}$and $\mathrm{CD} 8^{+} \mathrm{T}$ cell responses in mouse tumors expressing the corresponding antigens (154-156). Although these preclinical studies offer promise, specific viral antigens are still the immunodominant epitopes, inducing stronger immune reaction against OVs than the one against the tumor. A comprehensive analysis of the immunodominant epitopes of each OV family would reveal target epitopes that could be specifically mutated to reduce antiviral immunity. Oncolytic vaccines with specific mutations in the immunodominant epitopes of the virus would potentiate antitumor immunity by reducing antiviral immunity, resulting in a more efficient therapeutic approach that might be translated to clinical settings.

Combination of OVs with checkpoint inhibitors. The most promising strategy that has the potential to revolutionize treatment options is the combination of OVs with immune checkpoint inhibitors (157). Currently, the most widely studied immune checkpoints are cytotoxic T-lymphocyte associated protein-4 (CTLA-4), programmed death 1 (PD-1), and PD ligand 1 (PDL-1). The interaction between CTLA- 4 or PD-1 receptors on T lymphocytes and their ligands in tumor cells triggers an inhibitory signal that reduces proliferation of $\mathrm{CD}^{+} \mathrm{T}$ cells, resulting in immune tolerance of the tumor $(158,159)$. To overcome this dampened $\mathrm{T}$ cell response, CTLA-4 inhibitor (ipilimumab), PDL-1 inhibitors (avelumab, atezolizumab) or PD-1 inhibitors (lambrolizumab, pembrolizumab, nivolumab) have been tested in clinical trials $(160,161)$. Studies have shown that preexisting antitumor $\mathrm{T}$ cells in the TME predict favorable clinical responses to immune checkpoint inhibitors $(157,162)$. This evidence has led to the hypothesis that OV-mediated disruption of cancer cell immune tolerance could synergize with the response to checkpoint inhibitors (refs. 10, 163, 164, and Figure 3). In fact, OVs often induce IFN release in the TME, resulting in an upregulation of PDL-1 
expression on tumor cells (165). Previous studies have shown that the combination of reovirus and anti-PD-1 increased the ability of NK cells to kill reovirus-infected tumor cells, reduced immunosuppressive Tregs, and increased $\mathrm{CD}^{+} \mathrm{T}$ cells. This enhanced the antitumor immune response (166) and induced a robust memory response (10) in mouse tumor models. When combined with other viruses such as VSV or VV, a PDL-1 blockade also enhanced therapeutic outcomes in murine models of acute myeloid leukemia, colon cancer, and ovarian cancer $(164,167)$. Recent studies have shown that a triple combination of anti-CTLA-4, anti-PD-1, and oHSV-IL-12 resulted in longterm durable cures in most of the mice treated in two syngeneic models of GBM by inducing a profound increase in the ratio of $\mathrm{T}$ effector to Tregs (ref. 11 and Figure 3).

The first clinical trial combining T-VEC and anti-CTLA-4 demonstrated tolerable safety and objective responses compared with monotherapies in patients with advanced melanoma (ref. 168 and Table 1). The antitumor effect was observed in noninjected lesions as well as the injected ones, suggesting that the combination treatment induced a systemic effect. In another clinical trial, patients with advanced melanoma were treated intratumorally with coxsackievirus-21 in combination with anti-CTLA-4. The study showed strong evidence of synergistic antitumor effect, enhancing progression-free survival for greater than 6 months in patients who previously had progressed in response to anti-CTLA-4 monotherapies (ref. 169 and Table 1). Recently, a phase Ib clinical trial combining T-VEC with antiPD-1 has shown objective response rates (62\%) and complete response $(33 \%)$ in patients with metastatic melanoma (ref. 115 and Table 1). Furthermore, a systematic collection of sequential biopsies of injected and noninjected metastases obtained during different time points of treatment regimens showed an increase of $\mathrm{CD}^{+} \mathrm{T}$ cells and IFN- $\gamma$ expression in the majority of injected lesions as well as some noninjected lesions. A subsequent randomized phase III trial has just been completed and will be able to confirm these promising results (ClinicalTrials. gov NCT02263508 and Table 1).

\section{Conclusion and future perspectives}

OVs have been associated with a very favorable risk-benefit ratio and therefore offer a promising therapeutic option for cancer. In general, clinical studies performed thus far have demonstrated that OVs have a relatively tolerable toxicity in patients, with clinical trials reporting mild adverse events, few serious adverse events such as neurotoxicity, and minimal mortality(170). Although a number of exciting preclinical and clinical studies have indicated the strong potential of OVs, this strategy needs to be further improved for successful therapeutic efficacy in clinical settings for a broad spectrum of tumor types.
Intratumoral injection remains the most efficient and safest way to administer OVs. With systemic administration, neutralization in the bloodstream, virus sequestration, and nonspecific OV tropism to the tumor all reduce the number of OVs that reach the tumor. As such, different OV engineering strategies that extend OV circulation time after systemic administration should be continuously explored to increase antitumor efficacy. Cell-based carriers such as immune cells, SCs, and tumor cells have been shown to protect OVs from the immune system, support viral replication and amplification, and specifically target the virus to the tumor mass. In determining the most effective cell for OV delivery, it will be essential to consider the carrier cell's susceptibility to viral infection, replication, and release as well as its tumor-tracking ability. Developing more sophisticated mechanisms to repress viral replication in carrier cells and selectively reactivate OVs once carrier cells reach distant tumor foci will be critical in using carrier cells to deliver OVs to otherwise inaccessible tumors.

As more OVs progress toward clinical trials, having in-depth knowledge of the immune activation profile of each OV type will be crucial. Tumor biopsies and blood samples collected before and after treatment should be evaluated while planning for future clinical trials with viral vectors. Furthermore, patient selection will be an important consideration: immunocompromised patients may not be good candidates because OV-mediated antitumor immunity could be compromised in these patients. Clinical trials with immunotherapeutic OVs must be designed to consider that their antitumor efficacy requires priming and expansion of immune effector $\mathrm{CD}^{+} \mathrm{T}$ cells, migration to tumor sites, destruction of cancer cells, and induction of inflammation (76). Therefore, it is essential that clinical trials with immunotherapeutic OVs consider nontraditional end points to assess the benefit of OV treatments (171).

To conclude, OVTs offer tremendous promise for the treatment of cancer. Although patients who are refractory to the current standard of care may well benefit from this novel approach, eagerness to rush through clinical trials might jeopardize their health as well as the integrity of the OV field. Preclinical fervor should be tempered with caution during this precarious phase, and clinical trials should be carefully designed and have rigorous scientific backing.

\section{Acknowledgments}

This study was supported by funding from NIH-R01-CA204720 (to KS).

Address correspondence to: Khalid Shah, Brigham and Women's Hospital, Harvard Medical School, BTM 80160, 60 Fenwood Road, Boston, Massachusetts 02115, USA. Phone: 857.307.5233; Email:kshah@bwh.harvard.edu.
1. Chiocca EA, Rabkin SD. Oncolytic viruses and their application to cancer immunotherapy. Cancer Immunol Res. 2014;2(4):295-300.

2. Maroun J, Muñoz-Alía M, Ammayappan A, Schulze A, Peng KW, Russell S. Designing and building oncolytic viruses. Future Virol. 2017;12(4):193-213.

3. Guo ZS, et al. Oncolytic immunotherapy: con- ceptual evolution, current strategies, and future perspectives. Front Immunol. 2017;8:555.

4. Russell SJ, Barber GN. Oncolytic viruses as antigen-agnostic cancer vaccines. Cancer Cell. 2018;33(4):599-605.

5. Liu TC, Galanis E, Kirn D. Clinical trial results with oncolytic virotherapy: a century of prom- ise, a decade of progress. Nat Clin Pract Oncol. 2007;4(2):101-117.

6. Senzer NN, et al. Phase II clinical trial of a granulocyte-macrophage colony-stimulating factor-encoding, second-generation oncolytic herpesvirus in patients with unresectable metastatic melanoma. J Clin Oncol. 2009;27(34):5763-5771. 
7. Park $\mathrm{BH}$, et al. Use of a targeted oncolytic poxvirus, JX-594, in patients with refractory primary or metastatic liver cancer: a phase I trial. Lancet Oncol. 2008;9(6):533-542.

8. Andtbacka RH, et al. Talimogene laherparepvec improves durable response rate in patients with advanced melanoma. JClin Oncol. 2015;33(25):2780-2788

9. Liu BL, et al. ICP34.5 deleted herpes simplex virus with enhanced oncolytic, immune stimulating, and anti-tumour properties. Gene Ther. 2003;10(4):292-303.

10. Zamarin D, et al. Localized oncolytic virotherapy overcomes systemic tumor resistance to immune checkpoint blockade immunotherapy. Sci Transl Med. 2014;6(226):226ra32.

11. Saha D, Martuza RL, Rabkin SD. Macrophage polarization contributes to glioblastoma eradication by combination immunovirotherapy and immune checkpoint blockade. Cancer Cell. 2017;32(2):253-267.e5.

12. Kirn D. Clinical research results with dl1520 (Onyx-015), a replication-selective adenovirus for the treatment of cancer: what have we learned? Gene Ther. 2001;8(2):89-98.

13. Nemunaitis J, et al. A phase I trial of intravenous infusion of ONYX-015 and enbrel in solid tumor patients. Cancer Gene Ther. 2007;14(11):885-893.

14. Yaacov B, et al. Extracellular matrix constituents interfere with Newcastle disease virus spread in solid tissue and diminish its potential oncolytic activity. J Gen Virol. 2012;93(pt 8):1664-1672.

15. Wojton J, Kaur B. Impact of tumor microenvironment on oncolytic viral therapy. Cytokine Growth Factor Rev. 2010;21(2-3):127-134.

16. Guedan S, Rojas JJ, Gros A, Mercade E, Cascallo M, Alemany R. Hyaluronidase expression by an oncolytic adenovirus enhances its intratumoral spread and suppresses tumor growth. Mol Ther. 2010;18(7):1275-1283.

17. Shuster S, Frost GI, Csoka AB, Formby B, Stern R. Hyaluronidase reduces human breast cancer xenografts in SCID mice. Int J Cancer. 2002;102(2):192-197.

18. Martinez-Quintanilla J, He D, Wakimoto H, Alemany R, Shah K. Encapsulated stem cells loaded with hyaluronidase-expressing oncolytic virus for brain tumor therapy. Mol Ther. 2015;23(1):108-118.

19. Rodríguez-García A, et al. Safety and efficacy of VCN-01, an oncolytic adenovirus combining fiber HSG-binding domain replacement with RGD and hyaluronidase expression. Clin Cancer Res. 2015;21(6):1406-1418.

20. Martínez-Vélez N, et al. The oncolytic adenovirus VCN-01 as therapeutic approach against pediatric osteosarcoma. Clin Cancer Res. 2016;22(9):2217-2225.

21. Vera B, et al. Characterization of the antiglioma effect of the oncolytic adenovirus VCN-01.PLoS One. 2016;11(1):e0147211.

22. Schäfer S, et al. Vaccinia virus-mediated intra-tumoral expression of matrix metalloproteinase 9 enhances oncolysis of PC-3 xenograft tumors. BMC Cancer. 2012;12:366.

23. Kim JH, Lee YS, Kim H, Huang JH, Yoon AR, Yun CO. Relaxin expression from tumor-targeting adenoviruses and its intratumoral spread, apoptosis induction, and efficacy. J Natl Cancer Inst.
2006;98(20):1482-1493.

24. Jung $\mathrm{KH}$, et al. Oncolytic adenovirus expressing relaxin (YDC002) enhances therapeutic efficacy of gemcitabine against pancreatic cancer. Cancer Lett. 2017;396:155-166.

25. Lee SY, Park HR, Rhee J, Park YM, Kim SH. Therapeutic effect of oncolytic adenovirus expressing relaxin in radioresistant oral squamous cell carcinoma. Oncol Res. 2013;20(9):419-425.

26. Zhao H, et al. Oncolytic adenovirus, rAd.DCN, inhibits breast tumor growth lung metastasis in an immune-competent orthotopic xenograft model [published online ahead of print October 2, 2018]. Hum Gene Ther. https://doi. org/10.1089/hum.2018.055.

27. $\mathrm{Xu} \mathrm{W}$, et al. The systemic delivery of an oncolytic adenovirus expressing decorin inhibits bone metastasis in a mouse model of human prostate cancer. Gene Ther. 2015;22(3):247-256.

28. Gilkes DM, Semenza GL, Wirtz D. Hypoxia and the extracellular matrix: drivers of tumour metastasis. Nat Rev Cancer. 2014;14(6):430-439.

29. McAtee CO, Barycki JJ, Simpson MA. Emerging roles for hyaluronidase in cancer metastasis and therapy. Adv Cancer Res. 2014;123:1-34.

30. Ebert O, Shinozaki K, Kournioti C, Park MS, García-Sastre A, Woo SL. Syncytia induction enhances the oncolytic potential of vesicular stomatitis virus in virotherapy for cancer. Cancer Res. 2004;64(9):3265-3270.

31. Zhang J, Frolov I, Russell SJ. Gene therapy for malignant glioma using Sindbis vectors expressing a fusogenic membrane glycoprotein. JGene Med. 2004;6(10):1082-1091.

32. Guedan S, Gros A, Cascallo M, Vile R, Mercade E, Alemany R. Syncytia formation affects the yield and cytotoxicity of an adenovirus expressing a fusogenic glycoprotein at a late stage of replication. Gene Ther. 2008;15(17):1240-1245.

33. Gao Q, Lei T, Ye F. Therapeutic targeting of EGFR-activated metabolic pathways in glioblastoma. Expert Opin Investig Drugs. 2013;22(8):1023-1040.

34. Kim M, et al. Acquired resistance to reoviral oncolysis in Ras-transformed fibrosarcoma cells. Oncogene. 2007;26(28):4124-4134.

35. Kim YT, Ganly I, Brown R, Stuart D. Acquired resistance to cytolysis of the E1B-attenuated adenovirus, d11520, in ovarian tumour cell lines. Cancer Gene Ther. 2003;10(8):589-590.

36. Tong Y, et al. PI3K inhibitor LY294002 inhibits activation of the Akt/mTOR pathway induced by an oncolytic adenovirus expressing TRAIL and sensitizes multiple myeloma cells to the oncolytic virus. Oncol Rep. 2014;31(4):1581-1588.

37. Fu X, Tao L, Rivera A, Zhang X. Rapamycin enhances the activity of oncolytic herpes simplex virus against tumor cells that are resistant to virus replication. Int J Cancer. 2011;129(6):1503-1510.

38. Mérino D, Lalaoui N, Morizot A, Solary E, Micheau O. TRAIL in cancer therapy: present and future challenges. Expert Opin Ther Targets. 2007;11(10):1299-1314.

39. Tamura K, et al. Multimechanistic tumor targeted oncolytic virus overcomes resistance in brain tumors. Mol Ther. 2013;21(1):68-77.

40. Jahan N, Lee JM, Shah K, Wakimoto H. Therapeutic targeting of chemoresistant and recurrent glioblastoma stem cells with a proapoptotic variant of oncolytic herpes simplex virus. Int J Cancer. 2017;141(8):1671-1681.

41. Jo M, et al. Apoptosis induced in normal human hepatocytes by tumor necrosis factor-related apoptosis-inducing ligand. Nat Med. 2000;6(5):564-567.

42. Zaiss AK, Machado HB, Herschman HR. The influence of innate and pre-existing immunity on adenovirus therapy. JCell Biochem. 2009;108(4):778-790.

43. White CL, et al. Characterization of the adaptive and innate immune response to intravenous oncolytic reovirus (Dearing type 3 ) during a phase I clinical trial. Gene Ther. 2008;15(12):911-920.

44. Heo J, et al. Randomized dose-finding clinical trial of oncolytic immunotherapeutic vaccinia JX-594 in liver cancer. Nat Med. 2013;19(3):329-336.

45. Galanis E, et al. Phase I trial of intraperitoneal administration of an oncolytic measles virus strain engineered to express carcinoembryonic antigen for recurrent ovarian cancer. Cancer Res. 2010;70(3):875-882.

46. Shashkova EV, Doronin K, Senac JS, Barry MA. Macrophage depletion combined with anticoagulant therapy increases therapeutic window of systemic treatment with oncolytic adenovirus. Cancer Res. 2008;68(14):5896-5904.

47. Alemany R, Suzuki K, Curiel DT. Blood clearance rates of adenovirus type 5 in mice. J Gen Virol. 2000;81(Pt 11):2605-2609.

48. Qiao J, et al. Cyclophosphamide facilitates antitumor efficacy against subcutaneous tumors following intravenous delivery of reovirus. Clin Cancer Res. 2008;14(1):259-269.

49. Peng KW, et al. Using clinically approved cyclophosphamide regimens to control the humoral immune response to oncolytic viruses. Gene Ther. 2013;20(3):255-261.

50. Ni S, Bernt K, Gaggar A, Li ZY, Kiem HP, Lieber A. Evaluation of biodistribution and safety of adenovirus vectors containing group $\mathrm{B}$ fibers after intravenous injection into baboons. Hum Gene Ther. 2005;16(6):664-677.

51. Lech PJ, et al. Epitope dampening monotypic measles virus hemagglutinin glycoprotein results in resistance to cocktail of monoclonal antibodies. PLoS One. 2013;8(1):e52306.

52. Lech PJ, Pappoe R, Nakamura T, Russell SJ. Corrigendum to "Antibody neutralization of retargeted measles viruses" [Virology 454-455 (2014) 237-246]. Virology. 2014;464-465:460.

53. Fisher KD, Green NK, Hale A, Subr V, Ulbrich K, Seymour LW. Passive tumour targeting of polymer-coated adenovirus for cancer gene therapy. $J$ Drug Target. 2007;15(7-8):546-551.

54. Jung SJ, et al. Safety profiles and antitumor efficacy of oncolytic adenovirus coated with bioreducible polymer in the treatment of a CAR negative tumor model. Biomacromolecules. 2015;16(1):87-96.

55. Yotnda P, Davis AR, Hicks MJ, Templeton NS, Brenner MK, Benner MK. Liposomal enhancement of the antitumor activity of conditionally replication-competent adenoviral plasmids. $\mathrm{Mol}$ Ther. 2004;9(4):489-495.

56. Hecht JR, et al. A phase I/II trial of intratumoral endoscopic ultrasound injection of ONYX-015 with intravenous gemcitabine in unresectable pancreatic 
carcinoma. Clin Cancer Res. 2003;9(2):555-561.

57. Khuri FR, et al. a controlled trial of intratumora ONYX-015, a selectively-replicating adenovirus, in combination with cisplatin and 5-fluorouracil in patients with recurrent head and neck cancer. Nat Med. 2000;6(8):879-885.

58. Li X, et al. The efficacy of oncolytic adenovirus is mediated by $\mathrm{T}$-cell responses against virus and tumor in Syrian hamster model. Clin Cancer Res. 2017;23(1):239-249.

59. Yang Y, et al. Systemic delivery of an oncolytic adenovirus expressing decorin for the treatment of breast cancer bone metastases. Hum Gene Ther. 2015;26(12):813-825.

60. Breitbach CJ, et al. Intravenous delivery of a multi-mechanistic cancer-targeted oncolytic poxvirus in humans. Nature. 2011;477(7362):99-102.

61. Breitbach CJ, et al. Targeted inflammation during oncolytic virus therapy severely compromises tumor blood flow. Mol Ther. 2007;15(9):1686-1693.

62. Di Paolo NC. Recognition of human oncogenic viruses by host pattern-recognition receptors. Front Immunol. 2014;5:353.

63. Sonabend AM, et al. Biodistribution of an oncolytic adenovirus after intracranial injection in permissive animals: a comparative study of Syrian hamsters and cotton rats. Cancer Gene Ther. 2009;16(4):362-372.

64. Liu R, Martuza RL, Rabkin SD. Intracarotid delivery of oncolytic HSV vector G47Delta to metastatic breast cancer in the brain. Gene Ther. 2005;12(8):647-654.

65. Sze DY, Iagaru AH, Gambhir SS, De Haan HA, Reid TR. Response to intra-arterial oncolytic virotherapy with the herpes virus NV1020 evaluated by [18F] fluorodeoxyglucose positron emission tomography and computed tomography. Hum Gene Ther. 2012;23(1):91-97.

66. Lopez MV, et al. A tumor-stroma targeted oncolytic adenovirus replicated in human ovary cancer samples and inhibited growth of disseminated solid tumors in mice. Mol Ther. 2012;20(12):2222-2233.

67. Nakashima H, Kaur B, Chiocca EA. Directing systemic oncolytic viral delivery to tumors via carrier cells. Cytokine Growth Factor Rev. 2010;21(2-3):119-126.

68. Collet G, Grillon C, Nadim M, Kieda C. Trojan horse at cellular level for tumor gene therapies. Gene. 2013;525(2):208-216.

69. Stojdl DF, et al. VSV strains with defects in their ability to shutdown innate immunity are potent systemic anti-cancer agents. Cancer Cell. 2003;4(4):263-275.

70. Norton L, Massagué J. Is cancer a disease of self-seeding? Nat Med. 2006;12(8):875-878.

71. García-Castro J, et al. Tumor cells as cellular vehicles to deliver gene therapies to metastatic tumors. Cancer Gene Ther. 2005;12(4):341-349.

72. Raykov Z, Balboni G, Aprahamian M, Rommelaere J. Carrier cell-mediated delivery of oncolytic parvoviruses for targeting metastases. Int J Cancer. 2004;109(5):742-749.

73. Thalmeier K, Meissner P, Reisbach G, Falk M, Brechtel A, Dörmer P. Establishment of two permanent human bone marrow stromal cell lines with long-term post irradiation feeder capacity. Blood. 1994;83(7):1799-1807.
74. Munguia A, Ota T, Miest T, Russell SJ. Cell carriers to deliver oncolytic viruses to sites of myeloma tumor growth. Gene Ther. 2008;15(10):797-806.

75. Coukos G, et al. Use of carrier cells to deliver a replication-selective herpes simplex virus- 1 mutant for the intraperitoneal therapy of epithelial ovarian cancer. Clin Cancer Res. 1999;5(6):1523-1537.

76. Gajewski TF, Schreiber H, Fu YX. Innate and adaptive immune cells in the tumor microenvironment. Nat Immunol. 2013;14(10):1014-1022.

77. Qiao J, et al. Loading of oncolytic vesicular stomatitis virus onto antigen-specific $\mathrm{T}$ cells enhances the efficacy of adoptive T-cell therapy of tumors. Gene Ther. 2008;15(8):604-616.

78. Ilett EJ, et al. Dendritic cells and T cells deliver oncolytic reovirus for tumour killing despite pre-existing anti-viral immunity. Gene Ther. 2009;16(5):689-699.

79. Kanzaki A, et al. Antitumor efficacy of oncolytic herpes simplex virus adsorbed onto antigen-specific lymphocytes. Cancer Gene Ther. 2012;19(4):292-298.

80. Pfirschke C, Schirrmacher V. Cross-infection of tumor cells by contact with $\mathrm{T}$ lymphocytes loaded with Newcastle disease virus. Int JOncol. 2009;34(4):951-962.

81. VanSeggelen H, Tantalo DG, Afsahi A, Hammill JA, Bramson JL. Chimeric antigen receptor-engineered T cells as oncolytic virus carriers. $\mathrm{Mol}$ Ther Oncolytics. 2015;2:15014.

82. Qiao J, et al. Purging metastases in lymphoid organs using a combination of antigen-nonspecific adoptive $\mathrm{T}$ cell therapy, oncolytic virotherapy and immunotherapy. Nat Med.2008;14(1):37-44.

83. Willmon C, Harrington K, Kottke T, Prestwich R, Melcher A, Vile R. Cell carriers for oncolytic viruses: Fed Ex for cancer therapy. Mol Ther. 2009;17(10):1667-1676

84. Noy R, Pollard JW. Tumor-associated macrophages: from mechanisms to therapy. Immunity. 2014;41(1):49-61.

85. Sawa-Wejksza K, Kandefer-Szerszeń M. Tumor-associated macrophages as target for antitumor therapy. Arch Immunol Ther Exp (Warsz). 2018;66(2):97-111.

86. Peng KW, et al. Tumor-associated macrophages infiltrate plasmacytomas and can serve as cell carriers for oncolytic measles virotherapy of disseminated myeloma. Am J Hematol. 2009;84(7):401-407.

87. Muthana M, et al. Macrophage delivery of an oncolytic virus abolishes tumor regrowth and metastasis after chemotherapy or irradiation. Cancer Res. 2013;73(2):490-495.

88. Thirumala S, Goebel WS, Woods EJ. Manufacturing and banking of mesenchymal stem cells. Expert Opin Biol Ther. 2013;13(5):673-691.

89. Mader EK, et al. Optimizing patient derived mesenchymal stem cells as virus carriers for a phase I clinical trial in ovarian cancer. J Transl Med. 2013;11:20.

90. Kidd S, Spaeth E, Klopp A, Andreeff M, Hall B, Marini FC. The (in) auspicious role of mesenchymal stromal cells in cancer: be it friend or foe. Cytotherapy. 2008;10(7):657-667.

91. Sonabend AM, Ulasov IV, Tyler MA, Rivera AA, Mathis JM, Lesniak MS. Mesenchymal stem cells effectively deliver an oncolytic adenovirus to intra- cranial glioma. Stem Cells. 2008;26(3):831-841.

92. Hakkarainen T, et al. Human mesenchymal stem cells lack tumor tropism but enhance the antitumor activity of oncolytic adenoviruses in orthotopic lung and breast tumors. Hum Gene Ther. 2007;18(7):627-641.

93. Duebgen M, et al. Stem cells loaded with multimechanistic oncolytic herpes simplex virus variants for brain tumor therapy. J Natl Cancer Inst. 2014;106(6):dju090.

94. Lu X, Wang X, Nian H, Yang D, Wei R. Mesenchymal stem cells for treating autoimmune dacryoadenitis. Stem Cell Res Ther. 2017;8(1):126

95. Fujii S, et al. Immunological properties of neural crest cells derived from human induced pluripotent stem cells. Stem Cells Dev. 2019;28(1):28-43.

96. Sontag CJ, Nguyen HX, Kamei N, Uchida N, Anderson AJ, Cummings BJ. Immunosuppressants affect human neural stem cells in vitro but not in an in vivo model of spinal cord injury. Stem Cells Transl Med. 2013;2(10):731-744.

97. Moreno R, et al. Enhanced antitumor efficacy of oncolytic adenovirus-loaded menstrual blood-derived mesenchymal stem cells in combination with peripheral blood mononuclear cells. Mol Cancer Ther. 2019;18(1):127-138.

98. Chang SM, et al. Patterns of care for adults with newly diagnosed malignant glioma. JAMA. 2005;293(5):557-564.

99. Kauer TM, Figueiredo JL, Hingtgen S, Shah K. Encapsulated therapeutic stem cells implanted in the tumor resection cavity induce cell death in gliomas. Nat Neurosci. 2011;15(2):197-204.

100.Du W, et al. Stem cell-released oncolytic herpes simplex virus has therapeutic efficacy in brain metastatic melanomas. Proc Natl Acad Sci US A. 2017;114(30):E6157-E6165.

101. Morshed RA, et al. Analysis of glioblastoma tumor coverage by oncolytic virus-loaded neural stem cells using MRI-based tracking and histological reconstruction. Cancer Gene Ther. 2015;22(1):55-61.

102.Ahmed AU, et al. Neural stem cell-based cell carriers enhance therapeutic efficacy of an oncolytic adenovirus in an orthotopic mouse model of human glioblastoma. Mol Ther. 2011;19(9):1714-1726.

103. Bagó JR, et al. Tumor-homing cytotoxic human induced neural stem cells for cancer therapy. $\mathrm{Sci}$ Transl Med. 2017;9(375):eaah6510.

104. Herrlinger U, et al. Neural precursor cells for delivery of replication-conditional HSV-1 vectors to intracerebral gliomas. Mol Ther. 2000;1(4):347-357.

105. Josiah DT, Zhu D, Dreher F, Olson J, McFadden G, Caldas H. Adipose-derived stem cells as therapeutic delivery vehicles of an oncolytic virus for glioblastoma. Mol Ther. 2010;18(2):377-385.

106. Power AT, Bell JC. Taming the Trojan horse: optimizing dynamic carrier cell/oncolytic virus systems for cancer biotherapy. Gene Ther. 2008;15(10):772-779.

107. Kim CK, et al. N-acetylcysteine amide augments the therapeutic effect of neural stem cell-based antiglioma oncolytic virotherapy. Mol Ther. 2013;21(11):2063-2073.

108. Nakashima H, Chiocca EA. Switching a replication-defective adenoviral vector into a replication-competent, oncolytic adenovirus. J Virol. 
2014;88(1):345-353.

109.Martinez-Quintanilla J, Bhere D, Heidari P, He D, Mahmood U, Shah K. Therapeutic efficacy and fate of bimodal engineered stem cells in malignant brain tumors. Stem Cells. 2013;31(8):1706-1714.

110.Liu J, Ma J, Tang K, Huang B. Therapeutic use of tumor cell-derived extracellular vesicles. Methods Mol Biol. 2017;1660:433-440.

111. Ran L, et al. Delivery of oncolytic adenovirus into the nucleus of tumorigenic cells by tumor microparticles for virotherapy. Biomaterials. 2016;89:56-66

112. Nande R, Howard CM, Claudio PP. Ultrasound-mediated oncolytic virus delivery and uptake for increased therapeutic efficacy: state of art. Oncolytic Virother. 2015;4:193-205.

113. Okunaga $S$, et al. Ultrasound as a method to enhance antitumor ability of oncolytic herpes simplex virus for head and neck cancer. Cancer Gene Ther. 2015;22(3):163-168.

114. Carlisle R, et al. Enhanced tumor uptake and penetration of virotherapy using polymer stealthing and focused ultrasound. J Natl Cancer Inst . 2013;105(22):1701-1710

115. Ribas A, et al. Oncolytic virotherapy promotes intratumoral $\mathrm{T}$ cell infiltration and improves anti-PD-1 immunotherapy. Cell. 2017;170(6):1109-1119.e10

116. Bell JC, Ilkow CS. A viro-immunotherapy triple play for the treatment of glioblastoma. Cancer Cell. 2017;32(2):133-134.

117. Lichty BD, Breitbach CJ, Stojdl DF, Bell JC. Going viral with cancer immunotherapy. Nat Rev Cancer. 2014;14(8):559-567.

118. Kroemer G, Galluzzi L, Kepp O, Zitvogel L. Immunogenic cell death in cancer therapy. Annu Rev Immunol. 2013;31:51-72.

119. Gauvrit A, Brandler S, Sapede-Peroz C, Boisgerault N, Tangy F, Gregoire M. Measles virus induces oncolysis of mesothelioma cells and allows dendritic cells to cross-prime tumor-specific CD8 response. Cancer Res. 2008;68(12):4882-4892.

120. Guillerme JB, et al. Measles virus vaccine-infected tumor cells induce tumor antigen cross-presentation by human plasmacytoid dendritic cells. Clin Cancer Res. 2013;19(5):1147-1158.

121. Woller N, et al. Viral infection of tumors overcomes resistance to PD-1-immunotherapy by broadening neoantigenome-directed T-cell responses. Mol Ther. 2015;23(10):1630-1640.

122.Prestwich RJ, et al. Immune-mediated antitumor activity of reovirus is required for therapy and is independent of direct viral oncolysis and replication. Clin Cancer Res. 2009;15(13):4374-4381.

123. Ilkow CS, et al. Reciprocal cellular cross-talk within the tumor microenvironment promotes oncolytic virus activity. Nat Med.2015;21(5):530-536.

124.Arulanandam $\mathrm{R}$, et al. VEGF-mediated induction of PRD1-BF1/Blimp1 expression sensitizes tumor vasculature to oncolytic virus infection. Cancer Cell. 2015;28(2):210-224.

125. Achard C, Surendran A, Wedge ME, Ungerechts G, Bell J, Ilkow CS. Lighting a fire in the tumor microenvironment using oncolytic immunotherapy. EBioMedicine. 2018;31:17-24.

126. Hiraoka K, et al. Retroviral replicating vector-mediated gene therapy achieves long-term control of tumor recurrence and leads to durable anticancer immunity. Neuro-oncology.
2017;19(7):918-929.

127. Yagiz K, et al. A Retroviral replicating vector encoding cytosine deaminase and 5-FC induces immune memory in metastatic colorectal cancer models. Mol Ther Oncolytics. 2018;8:14-26.

128. Inoko K, et al. Therapeutic activity of retroviral replicating vector-mediated prodrug activator gene therapy for pancreatic cancer. Cancer Gene Ther. 2018;25(7-8):184-195.

129. Marelli G, Howells A, Lemoine NR, Wang Y. Oncolytic viral therapy and the immune system: a double-edged sword against cancer. Front Immunol. 2018;9:866.

130. Thorne SH. The role of GM-CSF in enhancing immunotherapy of cancer. Immunotherapy. 2013;5(8):817-819.

131. Burke JM, et al. A first in human phase 1 study of CG0070, a GM-CSF expressing oncolytic adenovirus, for the treatment of nonmuscle invasive bladder cancer. J Urol. 2012;188(6):2391-2397.

132. Kanerva A, et al. Antiviral and antitumor T-cell immunity in patients treated with GM-CSF-coding oncolytic adenovirus. Clin Cancer Res. 2013;19(10):2734-2744.

133. Breitbach CJ, Thorne SH, Bell JC, Kirn DH. Targeted and armed oncolytic poxviruses for cancer: the lead example of JX-594. Curr Pharm Biotechnol. 2012;13(9):1768-1772.

134. Kaufman HL, Kim DW, DeRaffele G, Mitcham J, Coffin RS, Kim-Schulze S. Local and distant immunity induced by intralesional vaccination with an oncolytic herpes virus encoding GM-CSF in patients with stage IIIc and IV melanoma. Ann Surg Oncol. 2010;17(3):718-730.

135. Poh A. First oncolytic viral therapy for melanoma Cancer Discov. 2016;6(1):6.

136. Coffin R. Interview with Robert Coffin, inventor of T-VEC: the first oncolytic immunotherapy approved for the treatment of cancer. Immunotherapy. 2016;8(2):103-106.

137. Hu JC, et al. A phase I study of OncoVEXGM-CSF, a second-generation oncolytic herpes simplex virus expressing granulocyte macrophage colony-stimulating factor. Clin Cancer Res. 2006;12(22):6737-6747

138. Andtbacka RH, et al. Cutaneous head and neck melanoma in OPTiM, a randomized phase 3 trial of talimogene laherparepvec versus granulocyte-macrophage colony-stimulating factor for the treatment of unresected stage IIIB/IIIC/IV melanoma. Head Neck. 2016;38(12):1752-1758.

139. Merrick AE, Ilett EJ, Melcher AA. JX-594, a targeted oncolytic poxvirus for the treatment of cancer. Curr Opin Investig Drugs. 2009;10(12):1372-1382.

140. Hwang TH, et al. A mechanistic proof-of-concept clinical trial with JX-594, a targeted multi-mechanistic oncolytic poxvirus, in patients with metastatic melanoma. Mol Ther. 2011;19(10):1913-1922.

141. Breitbach CJ, Moon A, Burke J, Hwang TH, Kirn DH. A phase 2, open-label, randomized study of pexa-vec (JX-594) administered by intratumoral injection in patients with unresectable primary hepatocellular carcinoma. Methods Mol Biol. 2015;1317:343-357.

142.Park SH, et al. Phase $1 \mathrm{~b}$ trial of biweekly intravenous pexa-vec (JX-594), an oncolytic and immunotherapeutic vaccinia virus in colorectal cancer. Mol Ther. 2015;23(9):1532-1540.
143. Li J, et al. Chemokine expression from oncolytic vaccinia virus enhances vaccine therapies of cancer. Mol Ther. 2011;19(4):650-657.

144.Lapteva N, et al. Targeting the intratumoral dendritic cells by the oncolytic adenoviral vaccine expressing RANTES elicits potent antitumor immunity. J Immunother. 2009;32(2):145-156.

145. Dempe S, et al. Antitumoral activity of parvovirus-mediated IL-2 and MCP-3/CCL7 delivery into human pancreatic cancer: implication of leucocyte recruitment. Cancer Immunol Immunother. 2012;61(11):2113-2123.

146.Li J, O’Malley M, Sampath P, Kalinski P, Bartlett DL, Thorne SH. Expression of CCL19 from oncolytic vaccinia enhances immunotherapeutic potential while maintaining oncolytic activity. Neoplasia. 2012;14(12):1115-1121.

147. Ye JF, Qi WX, Liu MY, Li Y. The combination of NK and CD8+T cells with CCL2O/IL15-armed oncolytic adenoviruses enhances the growth suppression of TERT-positive tumor cells. Cell Immunol. 2017;318:35-41.

148. Choi IK, et al. Oncolytic adenovirus co-expressing IL-12 and IL-18 improves tumor-specific immunity via differentiation of T cells expressing IL-12R $\beta 2$ or IL-18R $\alpha$. Gene Ther. 2011;18(9):898-909.

149. Lee YS, et al. Enhanced antitumor effect of oncolytic adenovirus expressing interleukin-12 and B7-1 in an immunocompetent murine model. Clin Cancer Res. 2006;12(19):5859-5868.

150. Antoszczyk S, et al. Treatment of orthotopic malignant peripheral nerve sheath tumors with oncolytic herpes simplex virus. Neuro-oncology. 2014;16(8):1057-1066.

151. Gaston DC, et al. Production of bioactive soluble interleukin-15 in complex with interleukin-15 receptor $\alpha$ from a conditionally-replicating oncolytic HSV-1. PLoS One. 2013;8(11):e81768.

152. Fajardo CA, et al. Oncolytic adenoviral delivery of an EGFR-targeting T-cell engager improves antitumor efficacy. Cancer Res. 2017;77(8):2052-2063.

153. Tysome JR, et al. A novel therapeutic regimen to eradicate established solid tumors with an effective induction of tumor-specific immunity. Clin Cancer Res. 2012;18(24):6679-6689.

154. Liao JB, Publicover J, Rose JK, DiMaio D. Single-dose, therapeutic vaccination of mice with vesicular stomatitis virus expressing human papillomavirus type $16 \mathrm{E} 7$ protein. Clin Vaccine Immunol. 2008;15(5):817-824.

155. Bridle BW, et al. Vesicular stomatitis virus as a novel cancer vaccine vector to prime antitumor immunity amenable to rapid boosting with adenovirus. Mol Ther. 2009;17(10):1814-1821.

156. Mulryan K, et al. Attenuated recombinant vaccinia virus expressing oncofetal antigen (tumor-associated antigen) 5T4 induces active therapy of established tumors. Mol Cancer Ther. 2002;1(12):1129-1137.

157. Hamid O, et al. Safety and tumor responses with lambrolizumab (anti-PD-1) in melanoma. $N$ Eng JMed. 2013;369(2):134-144.

158. Castellanos JR, et al. B7-H3 role in the immune landscape of cancer. Am JClin Exp Immunol. 2017;6(4):66-75.

159. Bellmunt J, Powles T, Vogelzang NJ. A review on the evolution of PD-1/PD-L1 immunotherapy for bladder cancer: the future is now. Cancer Treat 
Rev. 2017;54:58-67.

160. Weber J, et al. Adjuvant nivolumab versus ipilimumab in resected stage iii or iv melanoma. $N$ Engl JMed. 2017;377(19):1824-1835.

161. Overman MJ, et al. Nivolumab in patients with metastatic DNA mismatch repair-deficient or microsatellite instability-high colorectal cancer (CheckMate 142): an open-label, multicentre, phase 2 study. Lancet Oncol. 2017;18(9):1182-1191.

162.Le DT, et al. PD-1 blockade in tumors with mismatch-repair deficiency. $N$ Engl J Med. 2015;372(26):2509-2520.

163. Ilett E, et al. Prime-boost using separate oncolytic viruses in combination with checkpoint blockade improves anti-tumour therapy. Gene Ther. 2017;24(1):21-30.

164. Shen W, Patnaik MM, Ruiz A, Russell SJ, Peng
KW. Immunovirotherapy with vesicular stomatitis virus and PD-L1 blockade enhances therapeutic outcome in murine acute myeloid leukemia Blood. 2016;127(11):1449-1458.

165. Bellucci R, et al. Interferon- $\gamma$-induced activation of JAK1 and JAK2 suppresses tumor cell susceptibility to NK cells through upregulation of PD-L1 expression. Oncoimmunology. 2015;4(6):e1008824.

166. Rajani K, et al. Combination therapy with reovirus and anti-PD-1 blockade controls tumor growth through innate and adaptive immune responses. Mol Ther. 2016;24(1):166-174.

167. Liu Z, Ravindranathan R, Kalinski P, Guo ZS, Bartlett DL. Rational combination of oncolytic vaccinia virus and PD-L1 blockade works synergistically to enhance therapeutic efficacy. Nat
Commun. 2017;8:14754.

168. Puzanov I, et al. Talimogene laherparepvec in combination with ipilimumab in previously untreated, unresectable stage IIIB-IV melanoma. JClin Oncol. 2016;34(22):2619-2626.

169.Andtbacka RHI, et al. Final data from CALM: A phase II study of Coxsackievirus A21 (CVA21) oncolytic virus immunotherapy in patients with advanced melanoma. J Clin Oncol. 2015;33(suppl 15):9030.

170.Buijs PR, Verhagen JH, van Eijck CH, van den Hoogen BG. Oncolytic viruses: from bench to bedside with a focus on safety. Hum Vaccin Immunother. 2015;11(7):1573-1584.

171. Hales RK, et al. Assessing oncologic benefit in clinical trials of immunotherapy agents. Ann Oncol. 2010;21(10):1944-1951. 